\title{
Intracranial pressure- and cerebral perfusion pressure threshold-insults in relation to cerebral energy metabolism in aneurysmal subarachnoid hemorrhage
}

\author{
Teodor Svedung Wettervik ${ }^{1}$ - Anders Hånell ${ }^{1} \cdot$ Timothy Howells $^{1} \cdot$ Elisabeth Ronne-Engström ${ }^{1} \cdot$ Anders Lewén $^{1}$. \\ Per Enblad ${ }^{1}$
}

Received: 29 November 2021 / Accepted: 22 February 2022 / Published online: 1 March 2022

(c) The Author(s) 2022

\begin{abstract}
Background The aim was to investigate the association between intracranial pressure (ICP)- and cerebral perfusion pressure (CPP) threshold-insults in relation to cerebral energy metabolism and clinical outcome after aneurysmal subarachnoid hemorrhage (aSAH).

Methods In this retrospective study, 75 aSAH patients treated in the neurointensive care unit, Uppsala, Sweden, 2008-2018, with ICP and cerebral microdialysis (MD) monitoring were included. The first 10 days were divided into early (day 1-3), early vasospasm (day 4-6.5), and late vasospasm phase (day 6.5-10). The monitoring time (\%) of ICP insults (>20 mmHg and $>25 \mathrm{mmHg}$ ), CPP insults ( $<60 \mathrm{mmHg},<70 \mathrm{mmHg},<80 \mathrm{mmHg}$, and $<90 \mathrm{mmHg}$ ), and autoregulatory CPP optimum (CPPopt) insults $(\triangle \mathrm{CPPopt}=\mathrm{CPP}-\mathrm{CPPopt}<-10 \mathrm{mmHg}, \Delta \mathrm{CPPopt}>10 \mathrm{mmHg}$, and within the optimal interval $\Delta \mathrm{CPPopt} \pm 10 \mathrm{mmHg}$ ) were calculated in each phase.

Results Higher percent of ICP above the $20 \mathrm{mmHg}$ and $25 \mathrm{mmHg}$ thresholds correlated with lower MD-glucose and increased MD-lactate-pyruvate ratio (LPR), particularly in the vasospasm phases. Higher percentage of CPP below all four thresholds $(60 / 70 / 80 / / 90 \mathrm{mmHg}$ ) also correlated with a MD pattern of poor cerebral substrate supply (MD-LPR $>40$ and MD-pyruvate $<120 \mu \mathrm{M}$ ) in the vasospasm phase and higher burden of CPP below $60 \mathrm{mmHg}$ was independently associated with higher MD-LPR in the late vasospasm phase. Higher percentage of CPP deviation from CPPopt did not correlate with worse cerebral energy metabolism. Higher burden of CPP-insults below all fixed thresholds in both vasospasm phases were associated with worse clinical outcome. The percentage of ICP-insults and CPP close to CPPopt were not associated with clinical outcome.

Conclusions Keeping ICP below $20 \mathrm{mmHg}$ and CPP at least above $60 \mathrm{mmHg}$ may improve cerebral energy metabolism and clinical outcome.
\end{abstract}

Keywords Aneurysmal subarachnoid hemorrhage $\cdot$ Cerebral perfusion pressure $\cdot$ Intracranial pressure $\cdot$ Microdialysis $\cdot$ Neurointensive care

This article is part of the Topical Collection on Neurosurgical intensive care

This work has not been previously presented.

Teodor Svedung Wettervik

teodor.svedung-wettervik@neuro.uu.se

1 Department of Neuroscience, Section of Neurosurgery, Uppsala University, 75185 Uppsala, Sweden

\section{Introduction}

Intracranial pressure (ICP) and cerebral perfusions pressure (CPP) are the two main treatment variables in the neurointensive care (NIC) of patients with severe aneurysmal subarachnoid hemorrhage (aSAH). For aSAH, the NIC targets for these variables are in clinical practice often extrapolated from traumatic brain injury (TBI) protocols. The current guidelines for TBI suggest keeping ICP below $22 \mathrm{mmHg}$ and maintaining CPP between 60 and $70 \mathrm{mmHg}$ [3]. aSAH patients commonly exhibit intracranial hypertension and cerebral ischemia [26], but there are currently no established 
aSAH guideline recommendations for ICP and CPP [5, 8]. There is some support for extrapolating the targeted thresholds from TBI to aSAH, as ICP above $20 \mathrm{mmHg}$ seems similarly dangerous and associated with unfavorable clinical outcome in both brain injury conditions $[10,18,22,26$, 32]. However, whereas a CPP-target within $60-70 \mathrm{mmHg}$ is associated with favorable outcome in TBI [3, 27], CPP above $70 \mathrm{mmHg}$ and higher is associated with a reduced risk of clinical deterioration and higher chance of favorable outcome in aSAH $[22,26]$. In addition to potential diseasespecific-fixed target thresholds, findings in TBI indicate that continuous, dynamic, patient-specific CPP-targets based on the concurrently optimal autoregulatory status (CPPopt) may be beneficial [1, 24, 26, 27, 30]. Keeping CPP close to CPPopt is associated with better brain tissue oxygenation [14], cerebral energy metabolism [30], and clinical outcome $[1,24,27,30]$ in TBI. In aSAH, recent studies demonstrated that CPP close to CPPopt was associated with a reduced rate of cerebral ischemia [15], but not with better clinical outcome [26].

Altogether, aSAH is a different disease entity as compared with TBI. aSAH patients often exhibit pre-existing cerebrovascular disese predisposing for the cerebral aneurysm that has ruptured and the acute phase is particularly characterized by development of hydrocephalus and cerebral vasospasm. To better understand the effects of ICP- and CPP-insults on the cerebral environment in aSAH patients, there is a need for high-resolution multimodality studies. The primary aim of this study was to determine the association between ICPand CPP-threshold insults and cerebral energy metabolism, as assessed with the cerebral microdialysis, in aSAH. The secondary aim was to determine the association of these insults with clinical outcome.

\section{Methods}

\section{Patients}

Patients with aSAH admitted to the Department of Neurosurgery at the University Hospital in Uppsala, Sweden, 2008-2018, were eligible for this study. Out of 605 adult patients with SAH and intracranial pressure (ICP) monitoring, 194 SAH patients were monitored with cerebral microdialysis (MD). The first 10 days after ictus were divided into three phases (early phase, early vasospasm phase, and late vasospasm phase) for temporal analyses (see "Data acquisition and analyses" section) and those 75 patients who had ICP and MD monitoring data in all three phases and who did not develop total brain infarction the first 10 days were included in the study (Supplementary Fig. 1). This patient cohort was hence based on the subgroup of patients with MD monitoring from a larger patient population that was studied in a previous study on ICP-/CPP-thresholds in relation to outcome [26].

\section{Treatment protocol}

Patients were managed in accordance with our standardized treatment protocol, which has been described in detail in previous studies $[22,25,26]$. Briefly, our treatment goals were ICP $\leq 20 \mathrm{~mm} \mathrm{Hg}, \mathrm{CPP} \geq 60 \mathrm{~mm} \mathrm{Hg}$, systolic blood pressure $>100 \mathrm{~mm} \mathrm{Hg}, \mathrm{pO}_{2}>12 \mathrm{kPa}$, arterial glucose $5-10 \mathrm{mmol} / \mathrm{L}(\mathrm{mM})$, electrolytes within normal ranges, slight hypervolemia with 0 fluid balance after aneurysm occlusion, and body temperature $<38^{\circ} \mathrm{C}$.

Unconscious (Glasgow Coma Scale Motor score (GCS $\mathrm{M})<6$ ) patients were intubated and mechanically ventilated. Propofol was given for sedation and morphine for analgesia. Aneurysms were early occluded, by endovascular embolization or surgical clipping. An external ventricular drain (EVD) was inserted to monitor ICP in unconscious (GCS $\mathrm{M}<6$ ) patients. If ICP was above $20 \mathrm{mmHg}$, the EVD was opened at a drainage level of $15 \mathrm{mmHg}$. Higher ICP-thresholds such as $20 \mathrm{mmHg}$ for EVD-opening were sometimes used for patients with brain edema or intrventricular clots due to the risk of slit ventricles which may cause false ICP readings. Open EVDs were occasionally closed even in these patients during periods of nursing or wake-up tests. Thiopental infusion and/or decompressive craniectomy (DC) were last-tier treatments for refractory intracranial hypertension. Arterial blood pressure (ABP) was chiefly maintained with fluids and vasopressors were only used if ABP/CPP still remained below the target thresholds. Dobutamine was used as the first line therapy for inotropic support and norepinephrine was used as a second line therapy for further vasopressor support. Nimodipine was given to all patients after NIC admission. Delayed ischemic neurological deficits (DIND) were defined as a new-onset of focal neurological deficit or deterioration in consciousness, not explained by, e.g., hydrocephalus, re-bleeding, or meningitis. If there was no manifest cerebral infarction on computed tomography (CT), a HHH (hypertension, hypervolemia, and hemodilution)-therapy was initiated. HHH-therapy included supine position, colloid fluids to increase the intravascular volume using albumin and dextran solutions, and moderately elevated systolic blood pressure target above $140 \mathrm{mmHg}$ [9].

\section{Outcome}

Clinical outcome was evaluated according to the Extended Glasgow Outcome Scale (GOS-E) 12 months after ictus $[28,31]$, by trained personnel using structured telephone interviews. GOS-E has eight categories of outcome, from 
death (1) to upper good recovery (8). Clinical outcome was dichotomized as favorable/unfavorable (GOS-E 5-8/1-4).

\section{Data acquisition and analyses}

ICP was monitored with an EVD system (HanniSet, Xtrans, Smith Medical GmbH, Glasbrunn, Germany). ABP was monitored invasively in the radial artery at heart level. A distinction between open and closed EVD was not done in the data analysis, since ICP was continuously measured also when the system was open and the drainage level adjusted according to the measured values to obtain the prescribed ICP. Physiological data were collected at $100 \mathrm{~Hz}$ using the Odin software [11]. Pressure reactivity index (PRx) was calculated as the $5 \mathrm{~min}$ correlation of $10 \mathrm{~s}$ averages of ICP and MAP [6, 27]. CPPopt was calculated continuously, minuteby-minute, as the CPP in a U-shaped curve with the concurrently lowest PRx the last $4 \mathrm{~h}$ [1]. CPPopt values were available during $54 \%$ of the monitoring time the first 10 days for all patients and daily CPPopt values could be calculated for $90-96 \%$ of the patients, depending on the day.

Cerebral energy metabolism was monitored with the 70 High Cut-Off MD catheter with a membrane length of $10 \mathrm{~mm}$ and a membrane cut-off of $20 \mathrm{kDa}$ (M Dialysis AB, Stockholm, Sweden). It was highly encouraged, but not mandatory, to insert these at the same time as the EVD, when ICP monitoring was considered. The MD catheter was placed via a burr-hole, adjacent to the EVD in normalappearing brain tissue in the right frontal lobe. The MD was perfused by means of a microinjection pump (106 MD Pump, M Dialysis AB) at a rate of $0.3 \mu \mathrm{L} / \mathrm{min}$ with sterile artificial cerebrospinal fluid containing - $\mathrm{NaCl} 147 \mathrm{mmol} / \mathrm{L}$ (mM), $\mathrm{KCl} 2.7 \mathrm{mM}, \mathrm{CaCl}_{2} 1.2 \mathrm{mM}$, and $\mathrm{MgCl}_{2} 0.85 \mathrm{mM}$. Cerebral interstitial glucose, pyruvate, lactate, and urea were estimated hourly, using a CMA 600 analyzer or the ISCUSflex Microdialysis Analyzer (M Dialysis AB). The MD urea was monitored to validate catheter performance [20]. Total imprecision coefficient of variation was $<10 \%$ for all analytes.

Mean daily values for ICP, CPP, CPPopt, MD-glucose, MD-pyruvate, MD-lactate, and MD-lactate-pyruvate-ratio (LPR) were evaluated the first 10 days post-ictus for those with favorable and unfavorable outcome in the Odin software. The burden of ICP- and CPP-insults were similarly calculated during the first 10 days for those with favorable and unfavorable outcome. The burden of ICP-insults with fixed targeted thresholds was calculated as the percentage of monitoring time above (i) $20 \mathrm{mmHg}$ and (ii) $25 \mathrm{mmHg}$. The threshold at $20 \mathrm{mmHg}$ was chosen in accordance with our management protocol and $25 \mathrm{mmHg}$ was chosen as a "severe insult." The burden of CPP-insults was calculated as the percentage of monitoring time below (i) $60 \mathrm{mmHg}$, (ii) $70 \mathrm{mmHg}$, (iii) $80 \mathrm{mmHg}$, and (iv) $90 \mathrm{mmHg}$. The threshold at $60 \mathrm{mmHg}$ was chosen in accordance with our management protocol and the threshold was then increased stepwise with $10 \mathrm{mmHg}$ in accordance with a previous study [26]. The burden of autoregulatory CPPopt-insults was calculated as the difference between actual CPP and calculated CPPopt ( $\triangle \mathrm{CPPopt}$ ), and categorized as (i) hypoperfusion, $\Delta$ CPPopt $<-10 \mathrm{mmHg}$ and (ii) hyperperfusion, $\Delta$ CPPopt $>10 \mathrm{mmHg}$ and lastly (iii) optimal, $\Delta$ CPPopt within $\pm 10 \mathrm{mmHg}$.

Furthermore, the energy metabolic pattern was classified as "poor cerebral substrate supply" (MD-LPR $>40$ and concurrent MD-pyruvate $<120 \mu \mathrm{M}$ ) and "cerebral mitochondrial dysfunction" (MD-LPR $>40$ and concurrent MDpyruvate $>120 \mu \mathrm{M})$. The percent of these cerebral states was evaluated the first 10 days post-ictus for those with favorable and unfavorable outcome. The MD-LPR threshold of 40 for metabolic disturbances was chosen in accordance with the consensus statement 2014 [13]. The MD-pyruvate threshold of $120 \mu \mathrm{M}$ was chosen because this is the highest pyruvate value for ischemic and the lowest value for non-ischemic cerebral conditions according to previous studies [19, 23].

The 10-day period was divided into three phases - (i) early phase (day 1 to 3 ), (ii) early vasospasm phase (day 4 to 6.5 ), and (iii) late vasospasm phase (day 6.5 to 10 ). The vasospasm phase (day 4 to 10) was hence split into two equally long periods of time. Mean values and the percent of monitoring time above/below the thresholds mentioned above were calculated for each phase in the Odin software.

\section{Statistical analysis}

The analysis aimed primarily to determine the association of insults of ICP- and CPP-thresholds with cerebral energy metabolism and secondarily the association of these insults with clinical outcome.

Nominal, ordinal, and continuous variables were described as numbers or proportions, medians with interquartile range (IQR), and mean values with standard deviation (SD).

The association among ICP- (\% above $20 \mathrm{mmHg}$ and $25 \mathrm{mmHg}$ ), fixed CPP- (\% below $60 \mathrm{mmHg}, 70 \mathrm{mmHg}$, $80 \mathrm{mmHg}$, and $90 \mathrm{mmHg}$ ), and autoregulatory CPPoptthresholds $(\Delta$ CPPopt $<-10 \mathrm{mmHg}, \Delta$ CPPopt $>10 \mathrm{mmHg}$, and $\Delta$ CPPopt within the optimal $\pm 10 \mathrm{mmHg}$ ) with cerebral energy metabolism was evaluated with univariate analysis (Spearman) for all three phases. Multiple linear regression analyses were performed with MD-LPR as the dependent variable in all phases. Age and GCS M at admission were included as baseline explanatory variables together with the ICP- and CPP-insult that had the strongest association with MD-LPR in the univariate analyses. $\log _{10}$ transformation of MD-LPR was done to optimize the regressions due to the skewness of the data. 
The association between the ICP-, CPP-, and CPPoptinsults and the MD-variables with clinical outcome was evaluated with the Spearman's rank correlation test. No multiple logistic regression analyses for clinical outcome were done due to the limited number of patients with favorable outcome $(n=16)$.

A $p$-value $<0.05$ was considered statistically significant. The statistical analyses were performed in SPSS version 28 (IBM Corp, Armonk, NY, USA).

Table 1 Demography, admission status, treatments, and clinical outcome

\begin{tabular}{ll}
\hline Patients, $n(\%)$ & $75(100)$ \\
\hline Age, mean $( \pm \mathrm{SD})$ & $59 \pm 11$ \\
Male/female, $n(\%)$ & $19 / 56(25 / 75)$ \\
GCS M at admission, median (IQR) & $5(5-6)$ \\
WFNS grade IV-V/I-III, $n(\%)$ & $59 / 15(79 / 21)$ \\
Fisher grade, median (IQR) & $4(3-4)$ \\
Aneurysm location, anterior/posterior, $n(\%)$ & $61 / 14(81 / 19)$ \\
Embolization/clip ligation/no treatment, $n(\%)$ & $59 / 14 / 2(79 / 19 / 3)$ \\
DIND, $n(\%)$ & $20(27)$ \\
Thiopental, $n(\%)$ & $11(15)$ \\
DC, $n(\%)$ & $8(11)$ \\
GOS-E, median (IQR) & $2(3-4)$ \\
Mortality, $n(\%)$ & $17(24)$ \\
Favorable outcome, $n(\%)$ & $16(22)$ \\
\hline
\end{tabular}

$D C$ decompressive craniectomy, DIND delayed ischemic neurological deficit, GCS $M$ Glasgow Coma Scale Motor score, GOS-E Glasgow Outcome Scale-Extended, IQR interquartile range, WFNS World Federation of Neurosurgical Societies

\section{Results}

\section{Patients, admission variables, treatments, and clinical outcome}

Seventy-five patients were included. The majority were female $(75 \%)$, mean age was around 60 years old, and typically had severe injuries based on the World Federation of Neurosurgical Societies (WFNS) and Fisher grade (Table 1). The aneurysm was more often located in the anterior (81\%) than the posterior (19\%) circulation and most patients were treated with endovascular embolization $(79 \%)$ rather than clipping (19\%). One in four patients developed DIND. Fifteen percent of the patients were treated with thiopental and $11 \%$ with decompressive craniectomy. Thiopental was initiated on day 5 in mean (range 2-11) due to ICP-problems with smaller infarctions in 8 cases and generalized edema in 3 cases. DC surgery was performed on day 5 in mean (range 2-10) due to ICP-problems with media infarction in 6 cases and generalized edema in 2 cases. After 1 year, $24 \%$ of the patients were deceased and $22 \%$ had recovered favorably and $78 \%$ unfavorably.

\section{Cerebral physiological variables and insults the first 10 days after ictus}

The physiological variables and the insult burden are described in Figs. 1, 2, 3, and 4. Mean ICP was around $10 \mathrm{mmHg}$ and the percentage of ICP above 20 and $25 \mathrm{mmHg}$ was approximately 5 and $2.5 \%$, respectively (Fig. 1A-C). Mean CPP was around $70 \mathrm{mmHg}$ in the early course and gradually increased to $90 \mathrm{mmHg}$ in the late vasospasm phase (Fig. 2A-E). Correspondingly, the
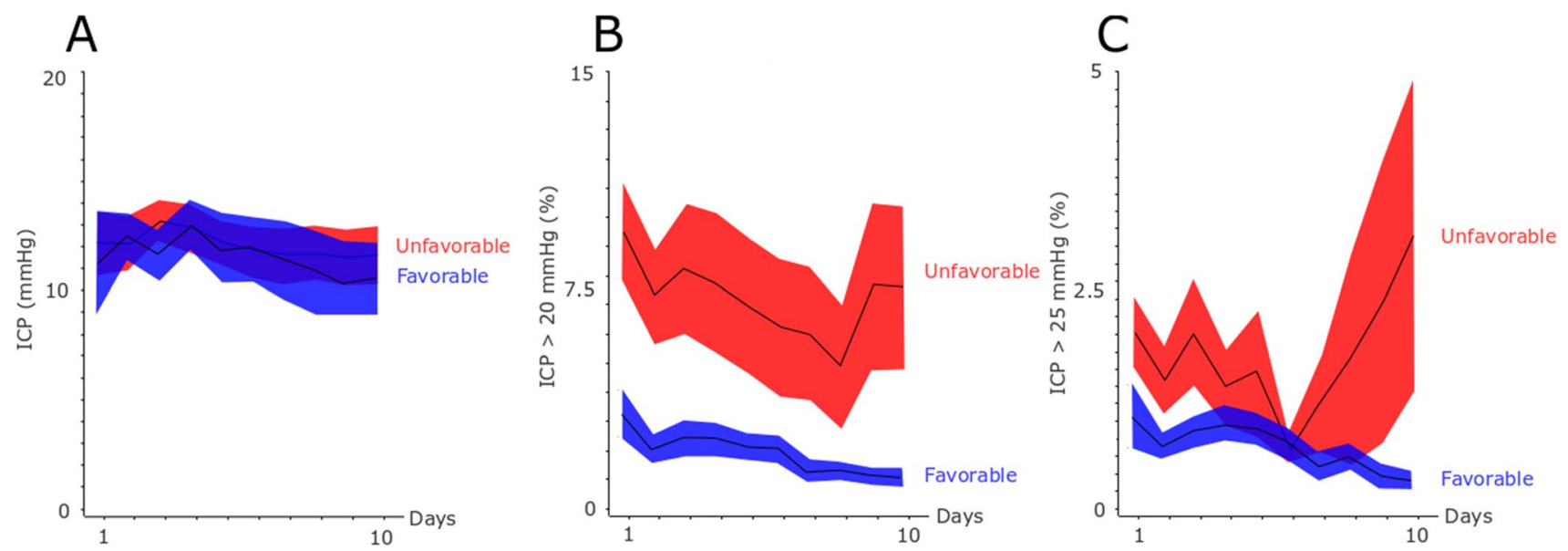

Fig. 1 A-C Mean ICP and ICP-insults with fixed thresholds above 20 and $25 \mathrm{mmHg}$ in relation to clinical outcome the first 10 days after ictus. The figure demonstrates mean daily values $(95 \% C I)$ of ICP
$\mathbf{A}$ and the percentage of ICP above $20 \mathrm{mmHg} \mathbf{B}$ and $25 \mathrm{mmHg} \mathbf{C}$ for those with favorable and unfavorable outcome the first 10 days after ictus. $C I$ confidence interval, ICP intracranial pressure 
Fig. 2 A-E Mean CPP and the burden of CPP-insults to different fixed thresholds in relation to clinical outcome the first 10 days after ictus.

The figure demonstrates mean daily values $(95 \% C I)$ of CPP $\mathrm{A}$ and the percentage of CPP below $60 \mathrm{mmHg} \mathrm{B}, 70 \mathrm{mmHg}$ $\mathbf{C}, 80 \mathrm{mmHg} \mathbf{D}$, and $90 \mathrm{mmHg}$ E for those with favorable and unfavorable outcome the first 10 days after ictus. $C I$ confidence interval, $C P P$ cerebral perfusion pressure
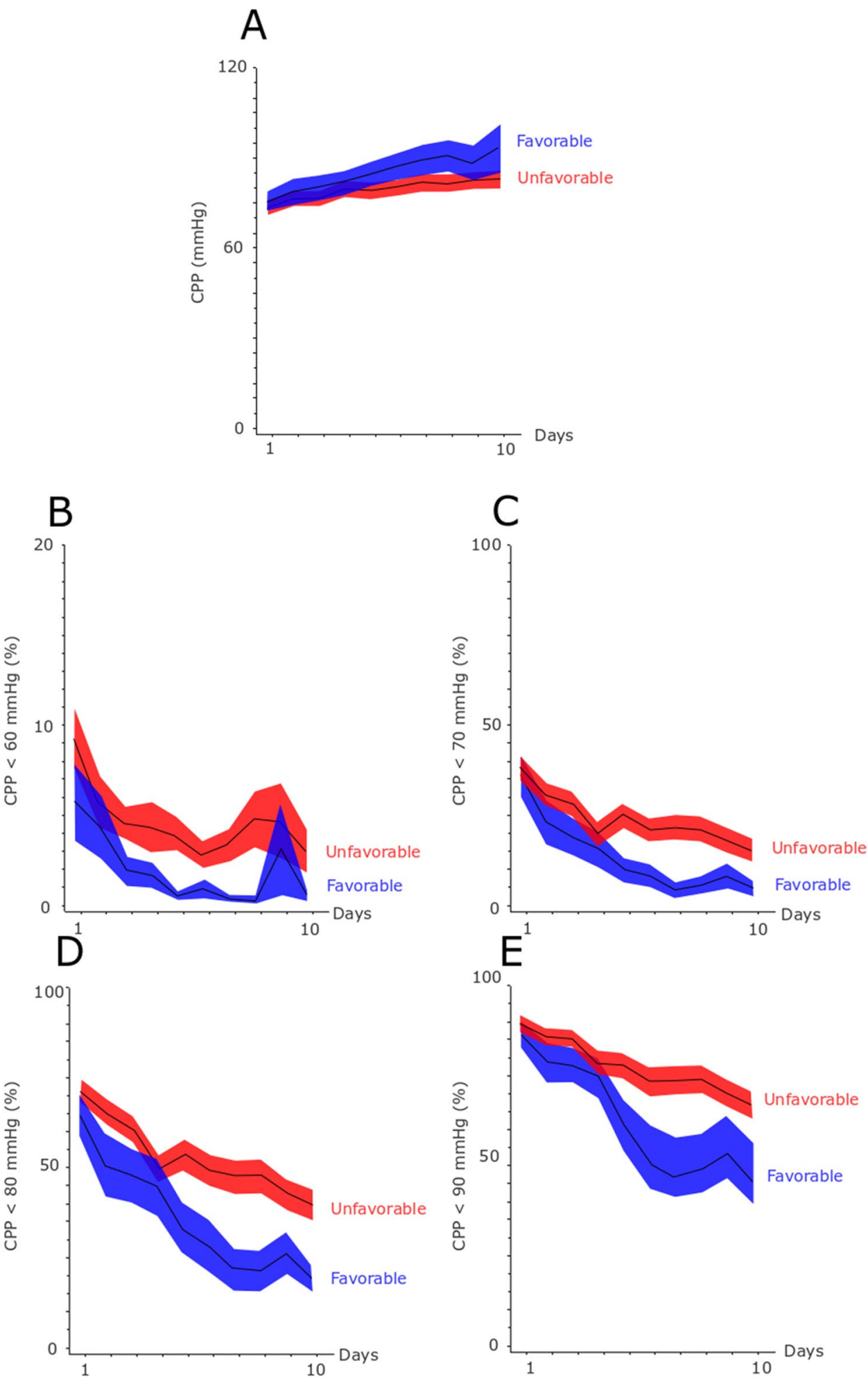

percent of CPP below $60 \mathrm{mmHg}, 70 \mathrm{mmHg}, 80 \mathrm{mmHg}$, and $90 \mathrm{mmHg}$ gradually decreased the first 10 days. CPPopt remained at approximately $80-90 \mathrm{mmHg}$ over the first 10 days (Fig. 3A-D). The percent of $\Delta$ CPPopt $<-10 \mathrm{mmHg}$ gradually decreased, whereas the percent of $\triangle \mathrm{CPPopt}>10 \mathrm{mmHg}$ increased during the first 10 days.

Mean MD-glucose was around $2 \mathrm{mM}$ and increased for those with favorable outcome, but decreased for those with unfavorable outcome in the late vasospasm phase 
Fig. 3 A-D Mean CPPopt and the burden of $\triangle$ CPPoptinsults in relation to clinical outcome the first 10 days after ictus. The figure demonstrates mean daily values $(95 \% C I)$ of CPPopt $\mathbf{A}$ and the percentage of $\triangle \mathrm{CPP}<-10 \mathrm{mmHg}$ $\mathbf{B}, \Delta \mathrm{CPP} \pm 10 \mathrm{mmHg} \mathbf{C}$, and $\Delta \mathrm{CPP}>10 \mathrm{mmHg} \mathbf{D}$ for those with favorable and unfavorable outcome the first 10 days after ictus. $C I$ confidence interval; $C P P$ cerebral perfusion pressure, CPPopt optimal cerebral perfusion pressure, $\triangle C P P C P P$ CPPopt
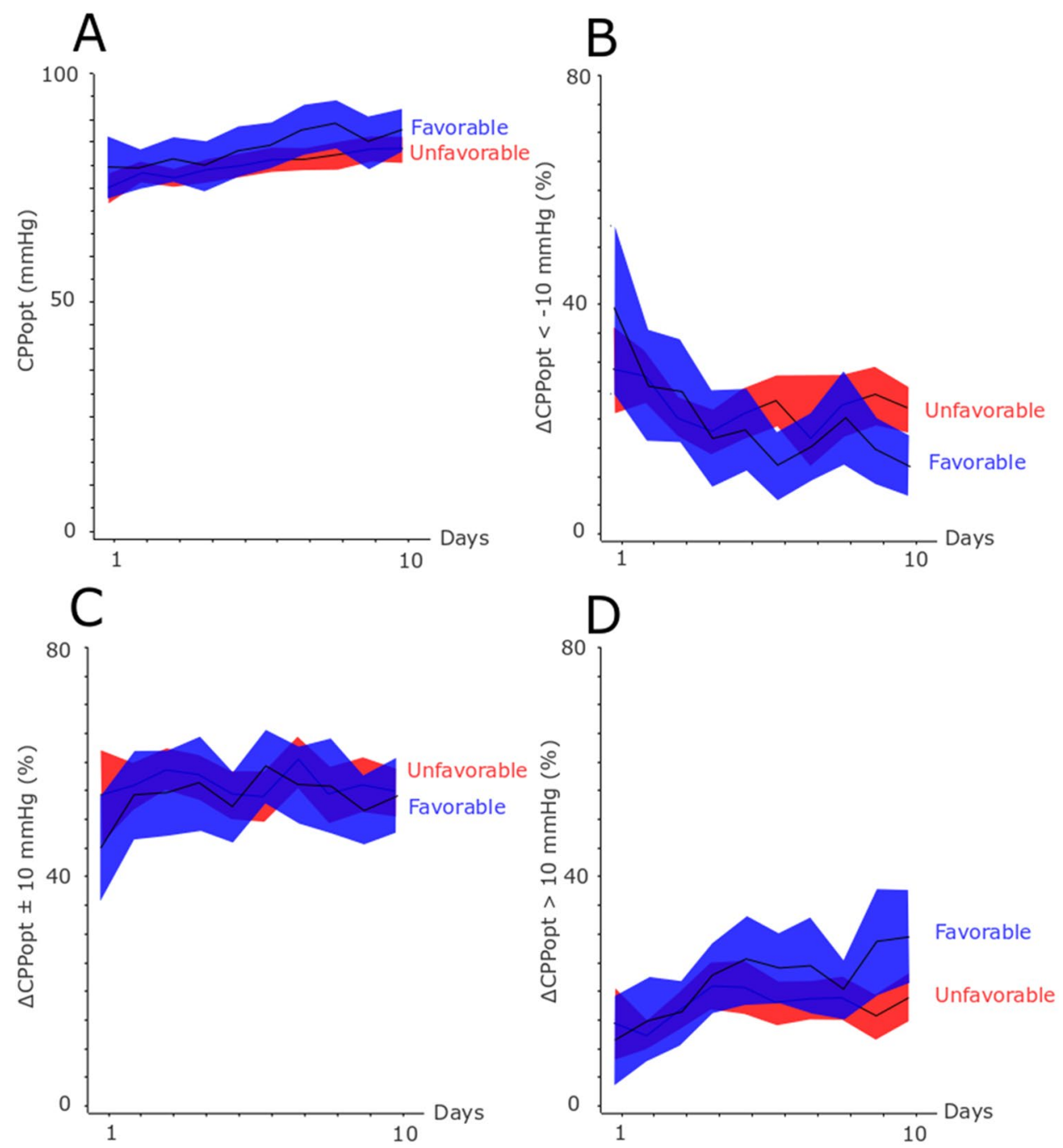

(Fig. 4A-F). MD-pyruvate was around $125 \mu \mathrm{M}$ at admission and increased to slightly above $200 \mu \mathrm{M}$ in the vasospasm phase. Mean MD-lactate was $4 \mathrm{mM}$ at admission and increased to $5 \mathrm{mM}$ in the late vasospasm phase. MD-LPR was mostly between 25 and 50. The percent of MD pattern indicative of poor cerebral substrate supply was around $25 \%$ in the early course and gradually decreased to 5-10\%. The pattern indicative of mitochondrial dysfunction increased slightly from $5 \%$ in the early phase to around $10 \%$ in the late vasospasm phase.

\section{Insults to ICP-, CPP-, and CPPopt-thresholds in relation to cerebral energy metabolism}

For fixed ICP-thresholds, in the early phase (Table 2), higher percent of ICP $>20 \mathrm{mmHg}$ correlated with a lower MD-pyruvate $(r=-0.27, p<0.05)$. In the early vasospasm phase, higher percent of ICP $>20 \mathrm{mmHg}$ was associated with lower MD-glucose $(r=-0.27, p<0.05)$ and higher MD-LPR $(r=0.23, p<0.05)$. Higher percent of
ICP $>25 \mathrm{mmHg}$ also correlated with a lower MD-glucose ( $r=-0.23, p<0.05$. In the late vasospasm phase, higher percent of ICP $>20 \mathrm{mmHg}$ was associated with lower MDglucose $(r=-0.27, p<0.05)$, higher MD-LPR $(r=0.25$, $p<0.05)$, increased burden of poor cerebral substrate supply $(r=0.37, p<0.001)$, and increased burden of mitochondrial dysfunction $(r=0.24, p<0.05)$. In the same phase, higher percent of ICP $>25 \mathrm{mmHg}$ was associated with increased MD-LPR $(r=0.26, p<0.05)$, increased burden of poor cerebral substrate supply ( $r=0.33, p<0.01)$, and increased burden of mitochondrial dysfunction $(r=0.24, p<0.05)$.

For fixed CPP-thresholds (Table 3), higher percent of CPP below $90 \mathrm{mmHg}$ correlated with lower MD-glucose $(r=-0.24, p<0.05)$ in the early phase and a higher percent of CPP below $60 \mathrm{mmHg}, 70 \mathrm{mmHg}, 80 \mathrm{mmHg}$, and $90 \mathrm{mmHg}$ all correlated with increased burden of poor cerebral substrate supply ( $r \approx 0.3$ and $p<0.05$ in all four correlations) in the late vasospasm phase.

For the autoregulatory CPP-thresholds (Table 4), a higher percent of $\triangle \mathrm{CPPopt} \pm 10 \mathrm{mmHg}$ correlated with higher 
MD-LPR $(r=0.26, p<0.05)$ and higher burden of poor cerebral substrate supply $(r=0.31, p<0.01)$ in the early phase. Higher percent of $\Delta \mathrm{CPPopt}>10 \mathrm{mmHg}$ was also associated with a lower burden of poor cerebral substrate supply $(r=-0.28, p<0.05)$ and lower burden of mitochondrial dysfunction $(r=-0.25, p<0.05)$ in the late vasospasm phase, but there was otherwise no association with cerebral energy metabolism.

In a multiple linear regression analyses (Table 5), a higher percent of $\mathrm{CPP}<60 \mathrm{mmHg}(\beta=0.24, p<0.05)$ and younger age $(\beta=-0.25, p<0.05)$ were independently associated with a higher MD-LPR ( $\log _{10}$ transformed) in the late vasospasm phase, whereas ICP $>25 \mathrm{mmHg}$ and GCS M at admission were not associatied with MD-LPR. In another regression, in which the autoregulatory thresholds $\Delta$ CPPopt $>10 \mathrm{mmHg}$ replaced the fixed CPP-threshold $\mathrm{CPP}<60 \mathrm{mmHg}$, deviation from the autoregulatory CPPthreshold was not associated with MD-LPR. Similar multiple linear regressions for MD-LPR in the early phase and the early vasospasm phase were not significant (data not shown).

\section{Insults to ICP-, CPP-, and CPPop-thresholds and MD-variables in relation to clinical outcome}

Insults above the ICP-thresholds $20 \mathrm{mmHg}$ and $25 \mathrm{mmHg}$ were not associated with clinical outcome (Table 2). Higher percent of CPP below $60 \mathrm{mmHg}, 70 \mathrm{mmHg}, 80 \mathrm{mmHg}$, and $90 \mathrm{mmHg}$ was associated with unfavorable outcome in both the early vasospasm phase and the late vasospasm phase (Table 3). Higher burden of CPP above CPPopt correlated with better outcome in both vasospasm phases, higher burden of CPP below CPPopt had the opposite association, and CPP close to CPPopt was not associated with clinical outcome (Table 4).

For the MD-variables, a higher MD-pyruvate in the early vasospasm phase and lower MD-LPR, lower burden of poor cerebral substrate supply, and lower burden of mitochondrial dysfunction in the late vasospasm phase correlated with more favorable outcome (higher GOS-E) (Supplementary Table 1).

\section{Discussion}

In the current study on 75 aSAH patients with ICP- and MD-monitoring, we found that ICP insults above $20 \mathrm{mmHg}$ and $25 \mathrm{mmHg}$ particularly in the vasospasm phases correlated with cerebral energy metabolic dysfunction, but not with clinical outcome. Furthermore, CPP below $60 \mathrm{mmHg}$ in the late vasospasm phase was independently associated with energy metabolic dysfunction and also correlated with worse clinical outcome. CPP close to CPPopt was not associated with better cerebral energy metabolism and clinical outcome. Our findings support that avoiding intracranial hypertension above $20 \mathrm{mmHg}$ and keeping CPP at least above $60 \mathrm{mmHg}$ may benefit cerebral energy metabolism and clinical outcome in aSAH.

\section{ICP-threshold insults in relation to cerebral energy metabolism and clinical outcome}

In univariate analyses, an increased burden of ICP-insults above 20 and $25 \mathrm{mmHg}$ correlated with worse cerebral energy metabolism, characterized by a lower MD-glucose, higher MD-LPR, and higher burden of both poor cerebral substrate supply and mitochondrial dysfunction, most pronounced in both vasospasm phases. Insults above the $20 \mathrm{mmHg}$ threshold rather than above $25 \mathrm{mmHg}$ generally correlated more strongly with an increased burden of poor cerebral substrate supply, possibly due to the low frequency of insults above $25 \mathrm{mmHg}$. An explanation for the associations between ICP insults and cerebral energy metabolic dysfunction could be that high ICP lowers CPP, leading to cerebral ischemia and poor substrate delivery. This is consistent with the fact that ICP-insults were not independently associated with increased MD-LPR in the multiple regressions. However, we cannot exclude the possibility that ICP itself may influence cerebral metabolism independently of CPP, although the effect may have been too small to be significant in the multiple regression analysis. It has for example been shown that high ICP increases capillary transit time heterogeneity which leads to disturbances in the cerebral microcirculation with shunting of blood and suboptimal delivery of energy substrates [33]. The fact that high ICP also correlated with mitochondrial dysfunction may also reflect that poor cerebral energy metabolism generates cerebral swelling and, per se, contributes to intracranial hypertension.

In the current study, there was no association between the burden of ICP insults above 20 or $25 \mathrm{mmHg}$ and clinical outcome. A potential explanation is that high ICP was actively treated and that we only included patients with $\mathrm{MD}$ monitoring the first 10 days, resulting in a small, homogeneous patient group that was for the most part severely injured. In previous studies from our group with larger aSAH populations, higher percent of ICP-insults above $25 \mathrm{~mm} \mathrm{Hg}$ was associated with clinical deterioration [22] and ICP-insults above $20 \mathrm{mmHg}$ with unfavorable outcome [26]. However, there are also some other studies which have not shown an association between ICP-insults and unfavorable outcome $[10,32]$. Furthermore, other approaches have been made to quantify the effects of intracranial hypertension. For example, the ICP dose, i.e., the combination of ICP intensity and the duration of that episode, has in one recent study been more strongly associated with unfavorable outcome than ICP above certain fixed thresholds [4]. On balance, despite the negative results in this paper, higher ICP for longer periods 

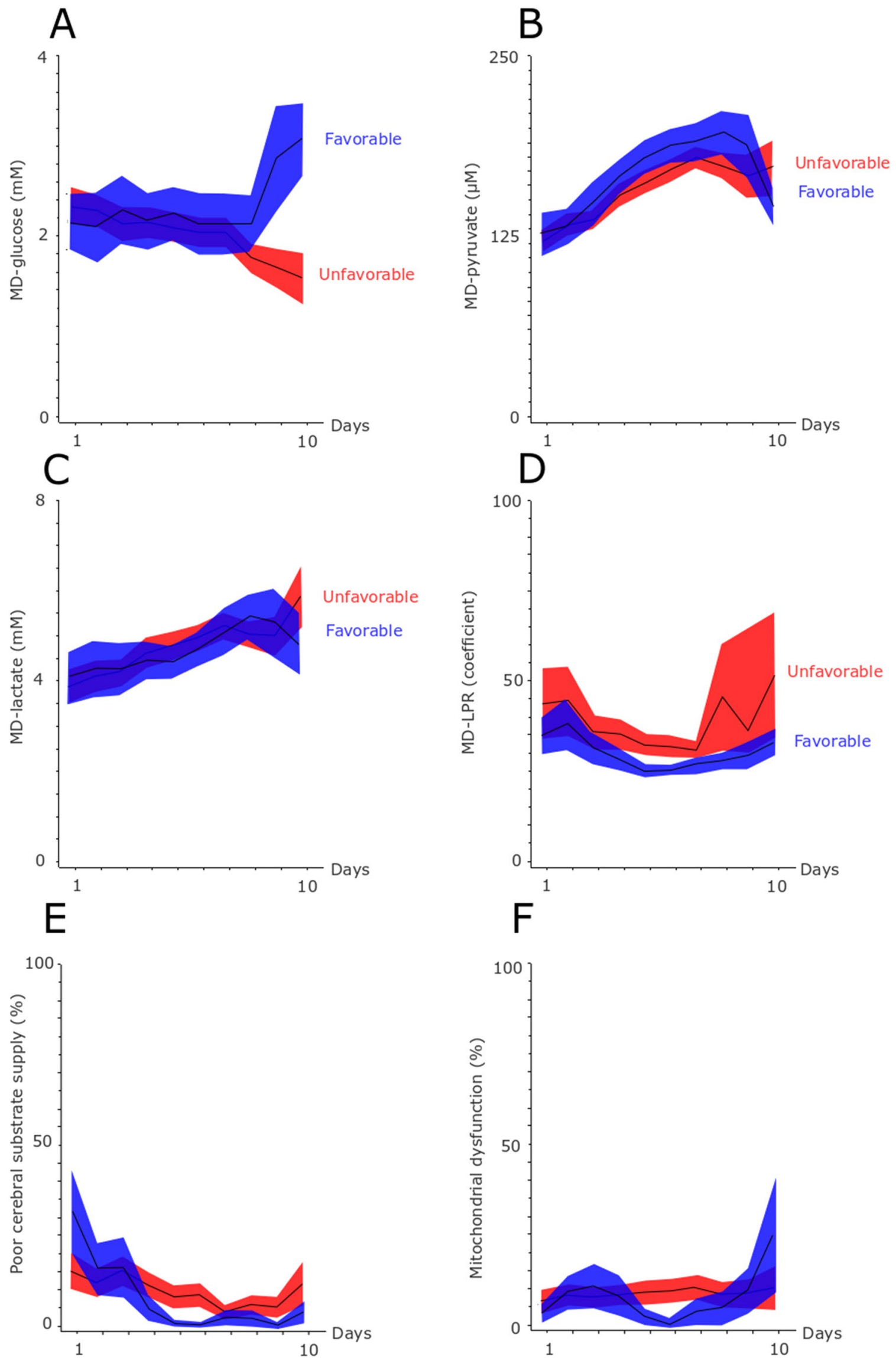

F

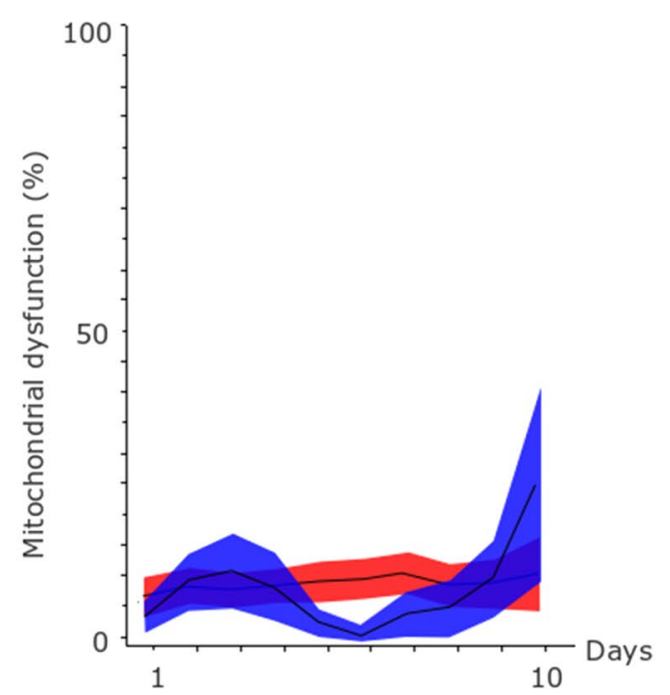


4Fig. 4 Cerebral energy metabolism in relation to clinical outcome the first 10 days after ictus. The figure demonstrates mean daily values (95\% CI) of MD-glucose A, MD-pyruvate B, MD-lactate C, MD-LPR D, and the burden (\%) of poor cerebral substrate supply $\mathbf{E}$ and mitochondrial dysfunction $\mathbf{F}$ for those with favorable and unfavorable outcome the first 10 days after ictus. Poor cerebral substrate supply was defined as MD-LPR $>40$ and concurrent MDpyruvate $<120 \mu \mathrm{M}$, whereas cerebral mitochondrial dysfunction was defined as MD-LPR $>40$ and concurrent MD-pyruvate $>120 \mu \mathrm{M}$. The MD-LPR threshold at 40 for metabolic disturbances was chosen in accordance with the consensus statement 2014 [13]. The MD-pyruvate threshold at $120 \mu \mathrm{M}$ was chosen as this is the highest pyruvate value for ischemic and the lowest value for non-ischemic cerebral conditions according to previous studies [19, 23]. $C I$ confidence interval, $L P R$ lactate-/pyruvate-ratio, $M D$ microdialysis

of time does seem to exert a negative effect on the brain and worsen outcome.

\section{CPP- and CPPopt-threshold insults in relation to cerebral energy metabolism and clinical outcome}

In this study, CPP below $60 \mathrm{mmHg}$ was indepently associated with a lower oxidative energy metabolism (higher LPR) and CPP below all four thresholds $(60 / 70 / 80 / 90 \mathrm{mmHg})$ were associated with an increased burden of poor cerebral substrate supply in the late vasospasm phase. CPP below $90 \mathrm{mmHg}$ was also slightly associated with a lower MD-glucose in the early phase. As a surrogate measure of CBF, CPP is flawed because it does not taking into account cerebrovascular resistance and reactivity. Intact cerebral autoregulation means that $\mathrm{CBF}$ remains constant over a wide range of CPP. Changes in CPP within these autoregulatory limits would not be expected to impact CBF and consequently cerebral energy metabolism. However, the cerebrovascular resistance rises in the vasospasm phase and the autoregulatory capacity is often disturbed after aSAH [17]. This increases the susceptibility for low CPP, leading to cerebral ischemia and poor cerebral substrate delivery, as was found in this study.
An earlier aSAH study on CBF by our group demonstrated that CPP values below CPPopt correlated with cerebral ischemia [15] and earlier TBI studies demonstrated that CPP deviations from CPPopt correlated with brain tissue hypoxia [14] and worse cerebral energy metabolism [30]. Unexpectedly, we did not find any association between $\triangle$ CPPopt-insults and worse cerebral energy metabolism. On the contrary, CPP within the suggested optimal interval $(\triangle \mathrm{CPPopt} \pm 10 \mathrm{mmHg})$ correlated with higher MD-LPR and higher burden of poor cerebral substrate supply in the early phase in univariate analyses. In addition, $\Delta$ CPPopt $>10 \mathrm{mmHg}$ correlated with lower burden of poor cerebral substrate supply and mitochondrial dysfunction in the late vasospasm phase, which rather indicates that high CPP was beneficial.

Furthermore, CPP deviation above CPPopt correlated with more favorable clinical outcome, CPP deviation below CPPopt had the opposite association, and CPP close to CPPopt had no association with clinical outcome. The interpretation of this is that CPPopt does not reflect "the optimal CPP-target" in aSAH, but rather that high CPP, per se, rather than close to CPPopt explained the association with better outcome. This is consistent with a previous larger study by our group, in which high CPP was beneficial but there was no association between the percent of CPP close to CPPopt and favorable outcome [26]. The negative findings for CPPopt in aSAH could be explained by that it is based on $\mathrm{PRx}$, which is a global measure of pressure autoregulation, but vasospasm, DIND, energy metabolic disturbances, and infarctions may be focal events which may go undetected by PRx [7]. Furthermore, we have earlier hypothesized that PRx may drift towards zero in case of distal cerebral vasospasm and may then falsely underestimate disturbed autoregulation [15]. Finally, CPPopt might be relatively high in case of cerebral vasospasm. It is possible that CPP values in these patients were typically not high enough to fully explore the upper limit of the CPPopt curve, which led to an underestimation of the true CPPopt.

Table 2 Fixed intracranial pressure targets in relation to cerebral energy metabolism and clinical outcome after aneurysmal subarachnoid hemorrhage — a Spearman's rank correlation analysis

\begin{tabular}{|c|c|c|c|c|c|c|}
\hline & \multicolumn{2}{|l|}{ Early phase } & \multicolumn{2}{|c|}{ Early vasospasm phase } & \multicolumn{2}{|c|}{ Late vasospasm phase } \\
\hline & $\mathrm{ICP}>20 \mathrm{mmHg}$ & $\mathrm{ICP}>25 \mathrm{mmHg}$ & $\mathrm{ICP}>20 \mathrm{mmHg}$ & $\mathrm{ICP}>25 \mathrm{mmHg}$ & $\mathrm{ICP}>20 \mathrm{mmHg}$ & $\mathrm{ICP}>25 \mathrm{mmHg}$ \\
\hline MD-glucose & -0.12 & -0.10 & $-0.27^{a}$ & $-0.23^{a}$ & $-0.27^{a}$ & -0.16 \\
\hline MD-pyruvate & $-0.27^{a}$ & -0.21 & -0.10 & -0.11 & -0.18 & -0.17 \\
\hline MD-lactate & -0.17 & -0.05 & 0.02 & 0.00 & 0.09 & 0.10 \\
\hline MD-LPR & 0.05 & 0.06 & $0.23^{a}$ & 0.20 & $0.25^{a}$ & $0.26^{a}$ \\
\hline Poor cerebral substrate supply & -0.00 & -0.01 & 0.06 & 0.02 & $0.37^{c}$ & $0.33^{b}$ \\
\hline Mitochondrial dysfunction & -0.08 & -0.07 & 0.14 & 0.16 & $0.24^{a}$ & $0.24^{a}$ \\
\hline GOS-E & -0.19 & -0.09 & -0.02 & -0.14 & -0.06 & -0.04 \\
\hline
\end{tabular}

${ }^{\mathrm{a}} p$-value $<0.05,{ }^{\mathrm{b}} p$-value $<0.01,{ }^{\mathrm{c}} p$-value $<0.001$. Bold and italics indicate statistical significance. GOS-E Glasgow Outcome Scale-Extended, $I C P$ intracranial pressure, $L P R$ lactate-pyruvate-ratio, $M D$ microdialysis 


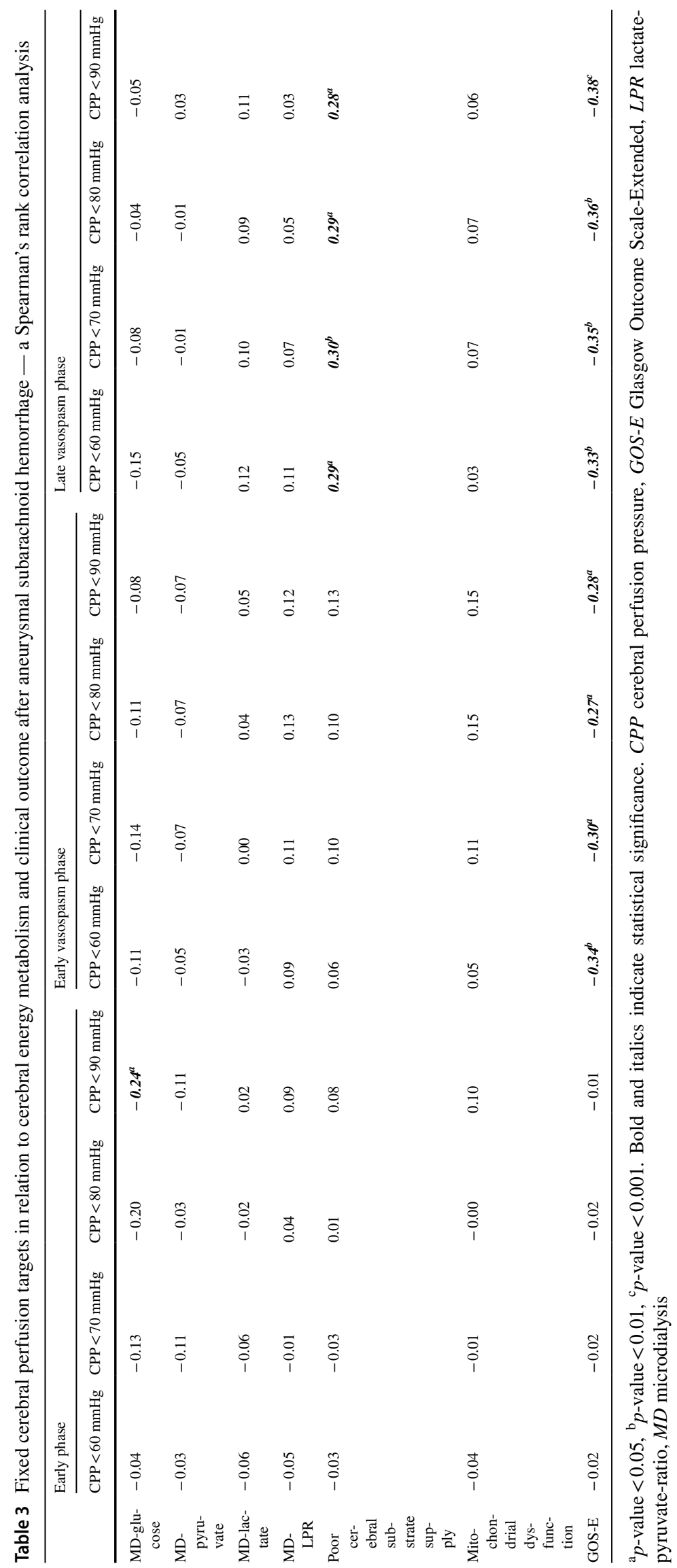




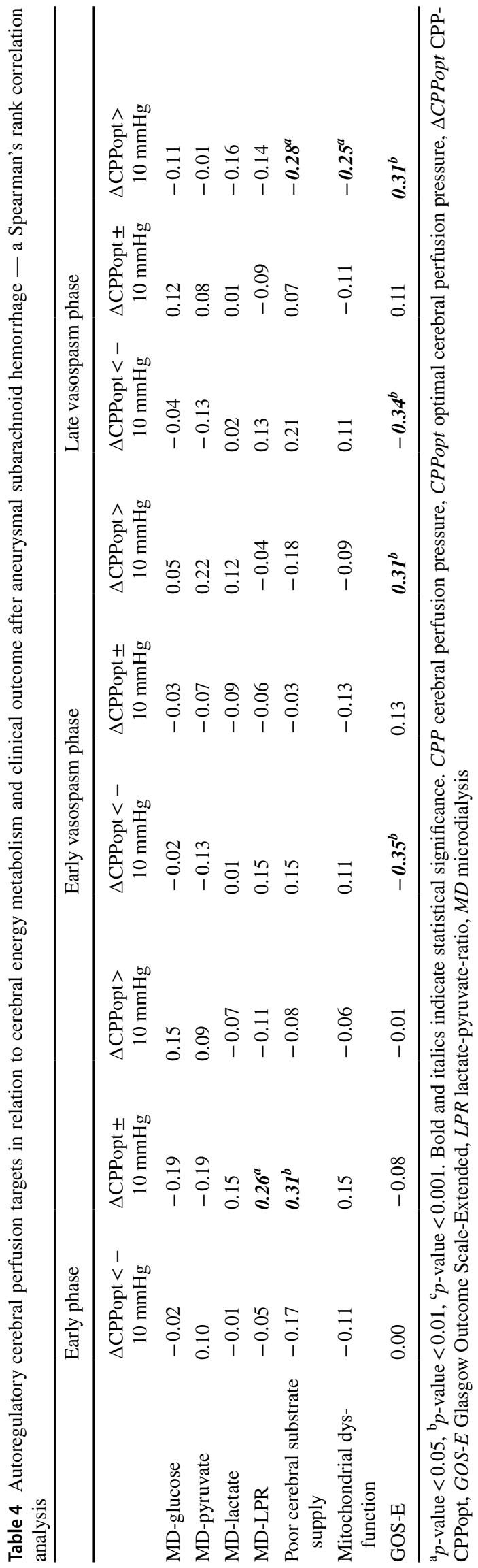


Table 5 Explanatory variables for lactate-pyruvate ratio in the late vasospasm phase in aneurysmal subarachnoid hemorrhage - a multiple linear regression analysis

\begin{tabular}{lll}
\hline Variables & $\beta(95 \%$ CI $)$ & $p$ \\
\hline Fixed CPP-thresholds & & \\
Age & $-0.25((-) 1.25-0.00)$ & $\mathbf{0 . 0 3}$ \\
GCS M at admission & $-0.05((-) 0.29-0.20)$ & 0.70 \\
CPP $<60 \mathrm{mmHg}$ & $0.24(0.00-0.49)$ & $\mathbf{0 . 0 5}$ \\
ICP $>25 \mathrm{mmHg}$ & $0.07((-) 0.13-0.26)$ & 0.60 \\
Autoregulatory CPP-thresholds & & \\
Age & $-0.22((-) 0.45-0.00)$ & 0.06 \\
GCS M at admission & $-0.10((-) 0.35-0.15)$ & 0.42 \\
$\Delta$ CPPopt $>10 \mathrm{mmHg}$ & $-0.19((-) 0.47-0.00)$ & 0.10 \\
ICP $>25 \mathrm{mmHg}$ & $0.14((-) 0.09-0.43)$ & 0.25 \\
\hline
\end{tabular}

Regression 1, fixed CPP-thresholds. $R^{2}=0.18$, ANOVA $p$-value $=0.009$. Regression 2, autoregulatory CPP-thresholds. $R^{2}=0.16$, ANOVA $p$-value $=0.016$. Due to skewness of LPR, a $\log _{10}$ transformation was done for this variable. Bold and italics indicate statistical significance. $C I$ confidence interval, $C P P$ cerebral perfusion pressure, CPPopt optimal CPP, GCS M Glasgow Coma Scale Motor score, ICP intracranial pressure, $\triangle C P P o p t$ CPP-CPPopt

Regarding the fixed targeted thresholds and clinical outcome, an increased burden of CPP below $60 \mathrm{mmHg}$, $70 \mathrm{mmHg}, 80 \mathrm{mmHg}$, and $90 \mathrm{mmHg}$ in the late vasospasm phase correlated both with lower burden of poor cerebral substrate supply and with worse clinical outcome. This is consistent with previous larger studies by our group [22, 26]. This supports the notion that spontaneuous CPP elevation up to $100 \mathrm{mmHg}$ should at least not be lowered. Our patients were only treated with inotropes or vasopressors to reach the lower CPP-threshold of $60 \mathrm{mmHg}$. Future studies are needed to determine if such agents should be used to target even higher CPP-values and if this would improve CBF, cerebral energy metabolism, and clinical outcome in aSAH.

Altogether, fixed rather than autoregulatory CPPthresholds seemed superior and CPP-targets at least above $60 \mathrm{mmHg}$, particularly in the vasospasm phase when the cerebrovascular resistance is increased, seems beneficial for cerebral energy metabolism and clinical outcome.

\section{Limitations}

First, the analysis of association between ICP- and CPPinsults in relation to clinical outcome needs to be interptreted with caution due to the limited number of patients and small proportion of cases with favorble outcome. Second, this was a single-center retrospective study based of patients with severe aSAH, which limits the external validity of our findings. Third, the validity of PRx and hence CPPopt has been questioned in case of an open EVD. However, we and others have found that ICP slow waves are preserved when the EVD is opened and PRx and CPPopt should then still be valid [2, 12]. Fourth, the MD measured cerebral energy metabolism in a focal area in normal-appearing brain tissue in the right frontal lobe in this study. In this focal area, several important pathomechanisms for energy metabolic disturbances may occur, related to, e.g., the initial injury insult, cortical spreading depolarization, oxygen diffusion limitations due to edema, focal vasospasm and autoregulatory disturbances, and mitochondrial dysfunction. Another MD location could have influenced the results, particularly between different vascular territories and in penumbral areas in proximity to an intracerebral hemorrhage [16, 29]. An earlier Xenon-CT-CBF and microdialysis study by our group showed that global cortical CBF correlated strongly with $\mathrm{CBF}$ around the microdialysis catheter placed in normalappearing brain [21]. It has also previously been demonstrated that disturbances in MD-variables (lactate, LPR, and glycerol) were more strongly correlated with clinical outcome when measured in normal-appearing brain rather than in perilesional areas [16]. This may also indicate that the energy metabolic state in the normal-appearing brain better reflects the global state of the brain, which is also more relevant for long-term recovery. However, the focality of MD is still limiting, when data are used to predict such a complex measure as clinical outcome that is influenced both by global and multifocal injuries in addition to many other variables such as patient age, co-morbidities, and neurorehabilitation. The correlation analyses between the MD and clinical outcome should hence be analyzed cautiously. Fifth, due to an absence of U-shaped curve between CPP and PRx, CPPopt could only be calculated during $54 \%$ of the monitoring time the first 10 days, similar to earlier findings in aSAH [26] and TBI [30]. This limits the validity of the CPPopt analyses. Fifth, many statistical tests were performed, which increases the risk of type I error. However, it is also possible that that some tests did not reach statistical significance due to limited number of patients. The significant correlations were also weak or moderate, which warrants caution when interpreting the data. However, the univariate correlations were only expected to be weak/moderate, considering the vast amount of variables affecting, e.g., LPR.

\section{Conclusions}

Keeping the intracranial pressure below $20 \mathrm{mmHg}$ and the cerebral perfusion pressure above at least $60 \mathrm{mmHg}$, and possibly considerably higher during the vasospasm phase, may be beneficial for cerebral energy metabolism and clinical outcome. Cerebral perfusion pressure close to the autoregulatory-oriented targets was not associated with better cerebral energy metabolism and clinical outcome. 
Supplementary Information The online version contains supplementary material available at https://doi.org/10.1007/s00701-022-05169-y.

Acknowledgements We express our gratitude to the personnel at the neurointensive-care unit, Uppsala University Hospital, for meticulous patient care.

Funding Open access funding provided by Uppsala University. The study was funded by the Department of Neuroscience, Uppsala University.

\section{Declarations}

Ethics approval All procedures performed in the studies involving humans were in accordance with the ethical standards of the national research committee and with the 1964 Helsinki Declaration and its later amendments. The study was approved by Uppsala University Regional Ethical Board (Dnr 2010/138 and Dnr 2010/138/1) and the Swedish Ethical Review Authority (2020-05462).

Informed consent Informed consent was obtained during NIC from the next of kin or by the patient after recovery at follow-up.

Conflict of interest The authors declare no competing interests.

Open Access This article is licensed under a Creative Commons Attribution 4.0 International License, which permits use, sharing, adaptation, distribution and reproduction in any medium or format, as long as you give appropriate credit to the original author(s) and the source, provide a link to the Creative Commons licence, and indicate if changes were made. The images or other third party material in this article are included in the article's Creative Commons licence, unless indicated otherwise in a credit line to the material. If material is not included in the article's Creative Commons licence and your intended use is not permitted by statutory regulation or exceeds the permitted use, you will need to obtain permission directly from the copyright holder. To view a copy of this licence, visit http://creativecommons.org/licenses/by/4.0/.

\section{References}

1. Aries MJ, Czosnyka M, Budohoski KP, Steiner LA, Lavinio A, Kolias AG, Hutchinson PJ, Brady KM, Menon DK, Pickard JD, Smielewski P (2012) Continuous determination of optimal cerebral perfusion pressure in traumatic brain injury. Crit Care Med 40:2456-2463. https://doi.org/10.1097/CCM.0b013e3182514eb6

2. Aries MJ, de Jong SF, van Dijk JM, Regtien J, Depreitere B, Czosnyka M, Smielewski P, Elting JW (2015) Observation of autoregulation indices during ventricular CSF drainage after aneurysmal subarachnoid hemorrhage: a pilot study. Neurocrit Care 23:347-354. https://doi.org/10.1007/s12028-015-0107-z

3. Carney N, Totten AM, O'Reilly C, Ullman JS, Hawryluk GW, Bell MJ, Bratton SL, Chesnut R, Harris OA, Kissoon N, Rubiano AM, Shutter L, Tasker RC, Vavilala MS, Wilberger J, Wright DW, Ghajar J (2017) Guidelines for the management of severe traumatic brain injury,fourth edition. Neurosurgery 80:6-15. https:// doi.org/10.1227/neu.0000000000001432

4. Carra G, Elli F, Ianosi B, Flechet M, Huber L, Rass V, Depreitere B, Güiza F, Meyfroidt G, Citerio G, Helbok R (2021) Association of dose of intracranial hypertension with outcome in subarachnoid hemorrhage. Neurocrit Care 34:722-730. https://doi.org/10.1007/ s12028-021-01221-4
5. Connolly ES Jr, Rabinstein AA, Carhuapoma JR, Derdeyn CP, Dion J, Higashida RT, Hoh BL, Kirkness CJ, Naidech AM, Ogilvy CS, Patel AB, Thompson BG, Vespa P (2012) Guidelines for the management of aneurysmal subarachnoid hemorrhage: a guideline for healthcare professionals from the American Heart Association/ American Stroke Association. Stroke 43:1711-1737. https://doi. org/10.1161/STR.0b013e3182587839

6. Czosnyka M, Smielewski P, Kirkpatrick P, Laing RJ, Menon D, Pickard JD (1997) Continuous assessment of the cerebral vasomotor reactivity in head injury. Neurosurgery 41:11-19

7. Davis SM, Andrews JT, Lichtenstein M, Rossiter SC, Kaye AH, Hopper J (1992) Correlations between cerebral arterial velocities, blood flow, and delayed ischemia after subarachnoid hemorrhage. Stroke 23:492-497. https://doi.org/10.1161/01.str.23.4.492

8. Diringer MN, Bleck TP, Claude Hemphill J, 3rd, Menon D, Shutter L, Vespa P, Bruder N, Connolly ES, Jr., Citerio G, Gress D, Hänggi D, Hoh BL, Lanzino G, Le Roux P, Rabinstein A, Schmutzhard E, Stocchetti N, Suarez JI, Treggiari M, Tseng MY, Vergouwen MD, Wolf S, Zipfel G (2011) Critical care management of patients following aneurysmal subarachnoid hemorrhage: recommendations from the Neurocritical Care Society's Multidisciplinary Consensus Conference. Neurocritical care 15:211-240. https://doi.org/10.1007/s12028-011-9605-9

9. Engquist H, Rostami E, Ronne-Engström E, Nilsson P, Lewén A, Enblad P (2018) Effect of HHH-therapy on regional CBF after severe subarachnoid hemorrhage studied by bedside Xenon-enhanced CT. Neurocrit Care 28:143-151. https://doi. org/10.1007/s12028-017-0439-y

10. Heuer GG, Smith MJ, Elliott JP, Winn HR, LeRoux PD (2004) Relationship between intracranial pressure and other clinical variables in patients with aneurysmal subarachnoid hemorrhage. J Neurosurg 101:408-416. https://doi.org/10.3171/jns. 2004.101.3.0408

11. Howells T, Elf K, Jones PA, Ronne-Engstrom E, Piper I, Nilsson P, Andrews P, Enblad P (2005) Pressure reactivity as a guide in the treatment of cerebral perfusion pressure in patients with brain trauma. J Neurosurg 102:311-317. https://doi.org/10. 3171/jns.2005.102.2.0311

12. Howells T, Johnson U, McKelvey T, Ronne-Engström E, Enblad P (2017) The effects of ventricular drainage on the intracranial pressure signal and the pressure reactivity index. J Clin Monit Comput 31:469-478. https://doi.org/10.1007/ s10877-016-9863-3

13. Hutchinson PJ, Jalloh I, Helmy A, Carpenter KL, Rostami E, Bellander BM, Boutelle MG, Chen JW, Claassen J, DahyotFizelier C, Enblad P, Gallagher CN, Helbok R, Hillered L, Le Roux PD, Magnoni S, Mangat HS, Menon DK, Nordstrom CH, O'Phelan KH, Oddo M, Perez Barcena J, Robertson C, Ronne-Engstrom E, Sahuquillo J, Smith M, Stocchetti N, Belli A, Carpenter TA, Coles JP, Czosnyka M, Dizdar N, Goodman JC, Gupta AK, Nielsen TH, Marklund N, Montcriol A, O'Connell MT, Poca MA, Sarrafzadeh A, Shannon RJ, SkjothRasmussen J, Smielewski P, Stover JF, Timofeev I, Vespa P, Zavala E, Ungerstedt U (2015) Consensus statement from the 2014 International Microdialysis Forum. Intensive Care Med 41:1517-1528. https://doi.org/10.1007/s00134-015-3930-y

14. Jaeger M, Dengl M, Meixensberger J, Schuhmann MU (2010) Effects of cerebrovascular pressure reactivity-guided optimization of cerebral perfusion pressure on brain tissue oxygenation after traumatic brain injury. Crit Care Med 38:1343-1347. https://doi.org/10.1097/CCM.0b013e3181d45530

15. Johnson U, Engquist H, Lewén A, Howells T, Nilsson P, RonneEngström E, Rostami E, Enblad P (2017) Increased risk of critical CBF levels in SAH patients with actual CPP below calculated optimal CPP. Acta Neurochir 159:1065-1071. https://doi. org/10.1007/s00701-017-3139-7 
16. Kofler M, Gaasch M, Rass V, Schiefecker AJ, Ianosi B, Lindner A, Beer R, Stover JF, Rhomberg P, Pfausler B, Thomé C, Schmutzhard E, Helbok R (2020) The importance of probe location for the interpretation of cerebral microdialysis data in subarachnoid hemorrhage patients. Neurocrit Care 32:135-144. https://doi.org/10.1007/s12028-019-00713-8

17. Lidington D, Wan H, Bolz SS (2021) Cerebral autoregulation in subarachnoid hemorrhage. Front Neurol 12:688362. https:// doi.org/10.3389/fneur.2021.688362

18. Magni F, Pozzi M, Rota M, Vargiolu A, Citerio G (2015) Highresolution intracranial pressure burden and outcome in subarachnoid hemorrhage. Stroke 46:2464-2469. https://doi.org/10. 1161/strokeaha.115.010219

19. Reinstrup P, Ståhl N, Mellergård P, Uski T, Ungerstedt U, Nordström C-H (2000) Intracerebral microdialysis in clinical practice: baseline values for chemical markers during wakefulness, anesthesia, and neurosurgery. Neurosurgery 47:701-710. https://doi.org/10.1097/00006123-200009000-00035

20. Ronne-Engström E, Cesarini KG, Enblad P, Hesselager G, Marklund N, Nilsson P, Salci K, Persson L, Hillered L (2001) Intracerebral microdialysis in neurointensive care: the use of urea as an endogenous reference compound. J Neurosurg 94:397-402. https://doi.org/10.3171/jns.2001.94.3.0397

21. Rostami E, Engquist H, Howells T, Ronne-Engström E, Nilsson P, Hillered LT, Lewén A, Enblad P (2017) The correlation between cerebral blood flow measured by bedside Xenon-CT and brain chemistry monitored by microdialysis in the acute phase following subarachnoid hemorrhage. Front Neurol 8:369. https://doi.org/10.3389/fneur.2017.00369

22. Ryttlefors M, Howells T, Nilsson P, Ronne-Engström E, Enblad $P$ (2007) Secondary insults in subarachnoid hemorrhage: occurrence and impact on outcome and clinical deterioration. Neurosurgery 61:704-714; discussion 714-705. https://doi.org/10. 1227/01.Neu.0000298898.38979.E3

23. Schulz MK, Wang LP, Tange M, Bjerre P (2000) Cerebral microdialysis monitoring: determination of normal and ischemic cerebral metabolisms in patients with aneurysmal subarachnoid hemorrhage. J Neurosurg 93:808-814. https://doi.org/10.3171/ jns.2000.93.5.0808

24. Steiner LA, Czosnyka M, Piechnik SK, Smielewski P, Chatfield D, Menon DK, Pickard JD (2002) Continuous monitoring of cerebrovascular pressure reactivity allows determination of optimal cerebral perfusion pressure in patients with traumatic brain injury. Crit Care Med 30:733-738. https://doi.org/10. 1097/00003246-200204000-00002

25. Svedung Wettervik T, Howells T, Hånell A, Ronne-Engström E, Lewén A, Enblad P (2021) Low intracranial pressure variability is associated with delayed cerebral ischemia and unfavorable outcome in aneurysmal subarachnoid hemorrhage. J Clin Monit Comput. https://doi.org/10.1007/s10877-021-00688-y

26. Svedung Wettervik T, Howells T, Lewén A, Ronne-Engström E, Enblad P (2021) Temporal dynamics of ICP, CPP, PRx, and CPPopt in high-grade aneurysmal subarachnoid hemorrhage and the relation to clinical outcome. Neurocrit Care. https://doi.org/ 10.1007/s12028-020-01162-4

27. Svedung Wettervik TM, Howells T, Enblad P, Lewén A (2019) Temporal neurophysiological dynamics in traumatic brain injury: role of pressure reactivity and optimal cerebral perfusion pressure for predicting outcome. J Neurotrauma 36:1818-1827. https://doi.org/10.1089/neu.2018.6157

28. Teasdale GM, Pettigrew LE, Wilson JT, Murray G, Jennett B (1998) Analyzing outcome of treatment of severe head injury: a review and update on advancing the use of the Glasgow Outcome Scale. J Neurotrauma 15:587-597. https://doi.org/10. 1089/neu.1998.15.587

29. Torné R, Culebras D, Sanchez-Etayo G, García-García S, Muñoz G, Llull L, Amaro S, Heering C, Blasco J, Zavala E, Enseñat J (2020) Double hemispheric microdialysis study in poor-grade SAH patients. Sci Rep 10:7466. https://doi.org/10. 1038/s41598-020-64543-x

30. Wettervik TS, Howells T, Hillered L, Rostami E, Lewén A, Enblad P (2021) Autoregulatory or fixed cerebral perfusion pressure targets in traumatic brain injury: determining which is better in an energy metabolic perspective. J Neurotrauma. https://doi.org/10.1089/neu.2020.7290

31. Wilson JL, Pettigrew LE, Teasdale GM (1998) Structured interviews for the Glasgow Outcome Scale and the extended Glasgow Outcome Scale: guidelines for their use. J Neurotrauma 15:573-585. https://doi.org/10.1089/neu.1998.15.573

32. Zoerle T, Lombardo A, Colombo A, Longhi L, Zanier ER, Rampini P, Stocchetti N (2015) Intracranial pressure after subarachnoid hemorrhage. Crit Care Med 43:168-176. https://doi. org/10.1097/ccm.0000000000000670

33. Østergaard L, Engedal TS, Aamand R, Mikkelsen R, Iversen NK, Anzabi M, Næss-Schmidt ET, Drasbek KR, Bay V, Blicher JU, Tietze A, Mikkelsen IK, Hansen B, Jespersen SN, Juul N, Sørensen JC, Rasmussen M (2014) Capillary transit time heterogeneity and flow-metabolism coupling after traumatic brain injury. J Cereb Blood Flow Metab 34:1585-1598. https://doi. org/10.1038/jcbfm.2014.131

Publisher's note Springer Nature remains neutral with regard to jurisdictional claims in published maps and institutional affiliations. 